\title{
FURTHER RESULTS ON SPECTRUM BLIND SAMPLING OF 2D SIGNALS
}

\author{
Raman Venkataramani and Yoram Bresler \\ Coordinated Science Laboratory \\ Department of Electrical and Computer Engineering \\ University of Illinois at Urbana-Champaign, Urbana, IL 61801
}

\begin{abstract}
We address the problem of sampling of 2D signals with sparse multi-band spectral structure. We show that the signal can be sampled at a fraction of the its Nyquist density determined by the occupancy of the signal in its frequency domain, but without explicit knowledge of its spectral structure. We find that such a signal can almost surely be reconstructed from its multi-coset samples provided that a universal pattern is used. Also, the scheme can attain the Landau-Nyquist minimum density asymptotically. The spectrum blind feature of our reconstruction scheme has potential applications in Fourier imaging. We apply the sampling scheme on a test image to demonstrate its performance.
\end{abstract}

\section{INTRODUCTION}

It is known that the sampling rate of any sampling scheme for a given class of multi-band signals is bounded from below by its Landau-Nyquist minimum rate, equal to the Lebesgue measure of the spectral suppose of the signal. The Nyquist rate, which is the smallest rate required to avoid aliasing when uniformly sampled, is much larger than the Landau-Nyquist rate in many cases. This is especially true for signals with poorly packable spectral supports. An example of such a signal in 2D is one whose spectral support is a thin annular region.

Our goal is to devise efficient sampling and reconstruction schemes for multi-band signals with their average sampling rates being as close to the theoretical minimum rates as possible. Furthermore, these schemes will be both universal and spectrum blind, allowing sampling and reconstruction of signals of a given "spectral occupancy" without prior knowledge of their spectral supports. The term spectral occupancy refers to the ratio of the measure of the spectral support to

Supported in part by the Joint Services Electronic Program Grant No. N00014-96-1-0129 and the National Science Foundation Grant No. MIP 97-07633 the measure of its smallest bounding box. The universality and spectrum-blind features are critical for applications such as Fourier imaging, where the "spectral support" corresponds to the support of an unknown object that is being imaged, and the samples are taken in the Fourier domain. In this paper we focus on the problem of $2 \mathrm{D}$ sampling.

\section{SAMPLING OF MULTI-BAND SIGNALS}

Cheung and Marks [1] showed that multi-band signals in $2 \mathrm{D}$ can be sampled and reconstructed at sub-Nyquist rates. Their idea was to first sample the signal at the Nyquist rate and then delete some samples periodically. Their argument is that, using knowledge of the spectral structure, the discarded samples can be recovered from those retained. This method admits sampling at rates that can be arbitrarily close to the minimum rate.

The simpler problem of 1D multi-band sampling has been addressed in $[2,3,4]$. The ideas in [3] are generalized to two and higher dimensions in [5]. Feng and Bresler [3, 5] introduced the idea of universal spectrum blind sampling. In this paper, we will provide an improvement over the the existing methods to achieve a lower sampling rate and/or improved robustness (conditioning) of the reconstruction, retaining universality and spectrum blindness. We shall employ multi-coset sampling for efficient representation of $2 \mathrm{D}$ multi-band signals and describe the algorithm for blind reconstruction.

\section{Multi-coset Sampling}

We will be concerned with the class of $2 \mathrm{D}$ continuous functions $x(\boldsymbol{t})$ of finite energy and whose Fourier transform has a known bounded support. Every such signal $x(\boldsymbol{t})$ can be perfectly reconstructed from its samples $x(\boldsymbol{n})$ at the Nyquist rate associated with the bounding box on its spectral support. If $x(\boldsymbol{n})$ can be perfectly recovered from its nonuniformly decimated version, then by acquiring, in the first place, only the samples re- 


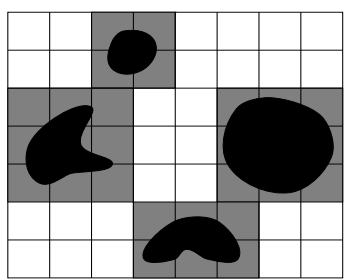

(a)

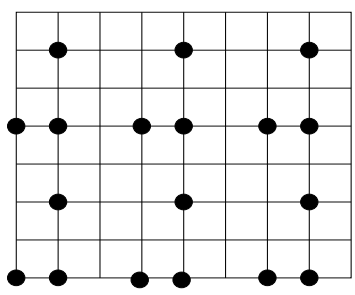

(b)
Figure 1: (a) Spectrum of a 2D multi-band signal. The spectral support is shown in black and the active cells in grey. (b) Multi-coset sampling consisting of 3 cosets out of a possible set of 12 .

tained in the decimation, the signal $x(\boldsymbol{t})$ can be perfectly recovered from its nonuniformly sampled version. Therefore, it is sufficient to focus our attention to discrete index signals.

Let $\mathcal{B}(\mathcal{F})$ denote the class of $2 \mathrm{D}$ discrete index multiband signals $x(\boldsymbol{n}), \boldsymbol{n} \in \mathrm{Z}^{2}$ with finite energy and spectral support $\mathcal{F}$, i.e. $\mathcal{B}(\mathcal{F})=\{x(\boldsymbol{n}): X(\boldsymbol{f})=0, \boldsymbol{f} \notin \mathcal{F}\}$ where $X(\boldsymbol{f})$ is the Fourier transform of $x(\boldsymbol{n})$ :

$$
X(f)=\sum_{Z^{2}} x(\boldsymbol{n}) \exp \left(-j 2 \pi \boldsymbol{f}^{T} \boldsymbol{n}\right)
$$

The spectral support $\mathcal{F}$ is assumed to be a finite union of connected regions in $[0,1]^{2}$. An example of a $2 \mathrm{D}$ multi-band signal is shown in Figure 1a. We may describe multi-coset sampling in the following way. Given two positive integers $L_{1}$ and $L_{2}$, we sample the $x(\boldsymbol{n})$ non-uniformly at the positions indicated by the following set of vectors in $\mathrm{Z}^{2}$ :

$$
\boldsymbol{\Psi}=\left\{\boldsymbol{L} \boldsymbol{m}+\boldsymbol{c}_{i}: \boldsymbol{m} \in \mathrm{Z}^{2}, i=1, \ldots, p\right\}
$$

where $\boldsymbol{L}=\operatorname{diag}\left(L_{1}, L_{2}\right)$ and $\left\{\boldsymbol{c}_{i}\right\}$ are $p \leq L_{1} L_{2}$ distinct vectors chosen from the set

$$
\mathcal{N}\left(L_{1}, L_{2}\right)=\left\{0, \ldots, L_{1}-1\right\} \times\left\{0, \ldots, L_{2}-1\right\}
$$

This is illustrated in Figure 1b. The set $\left\{\boldsymbol{c}_{i}\right\}$ constitutes the "sampling pattern" and the average sampling rate of the above scheme is $\frac{p}{L_{1} L_{2}}$. For convenience we define the following decimated $2 \mathrm{D}$ sequences:

$$
x_{i}(\boldsymbol{m})=x\left(\boldsymbol{L} \boldsymbol{m}+\boldsymbol{c}_{i}\right), \boldsymbol{m} \in \mathrm{Z}^{2}
$$

These sequences contain the samples taken on the $p$ cosets that make up the set $\boldsymbol{\Psi}$. The following section describes how the reconstruction from samples is accomplished.

\section{RECONSTRUCTION FOR KNOWN $\mathcal{F}$}

We shall partition the set $[0,1]^{2}$ (one period of the $2 \mathrm{D}$ spectrum $X(\boldsymbol{f}))$ into $L_{1} \times L_{2}$ smaller cells of size $\frac{1}{L_{1}} \times$ $\frac{1}{L_{2}}$. Denote these "spectral cells" by $\mathcal{C}_{l_{i}}$ for each $\boldsymbol{l}_{i} \in$ $\mathcal{N}\left(L_{1}, L_{2}\right), i=1, \ldots, L_{1} L_{2}$ :

$$
\mathcal{C}_{i}=\mathcal{C}_{\mathbf{0}}+\boldsymbol{L}^{-1} \boldsymbol{l}_{i}, \quad \mathcal{C}_{\mathbf{0}}=\left[0, \frac{1}{L_{1}}\right] \times\left[0, \frac{1}{L_{2}}\right]
$$

In other words, $\mathcal{C}_{l_{i}}$ is obtained by translating $\mathcal{C}_{\mathbf{0}}$ by $\boldsymbol{L}^{-1} \boldsymbol{l}_{i}$. The cell $\mathcal{C l}_{i}$ is termed "active" if $\mathcal{C l}_{i} \cap \mathcal{F} \neq \emptyset$. Let the active cells be specified by the following set of vectors

$$
\begin{aligned}
\mathcal{K} & =\left\{\boldsymbol{l} \in \mathcal{N}\left(L_{1}, L_{2}\right): \mathcal{C} 1 \cap \mathcal{F} \neq \emptyset\right\} \\
& \equiv\left\{\boldsymbol{k}_{1}, \boldsymbol{k}_{2}, \ldots, \boldsymbol{k}_{q}\right\} \subset \mathcal{N}\left(L_{1}, L_{2}\right)
\end{aligned}
$$

which we shall call the "spectral index set." The spectral occupancy $\Omega \mathrm{L}=\frac{q}{L_{1} L_{2}}$ of $\mathcal{F}$ at resolution $\left(L_{1}, L_{2}\right)$ is bounded from below by the Lebesgue measure of $\mathcal{F}$. Define vectors

$$
\boldsymbol{z}(\boldsymbol{f})=\left[X\left(\boldsymbol{f}+\boldsymbol{L}^{-1} \boldsymbol{k}_{1}\right), \ldots, X\left(\boldsymbol{f}+\boldsymbol{L}^{-1} \boldsymbol{k}_{q}\right)\right]^{T}
$$

consisting of the pieces of the active spectrum of $X(\boldsymbol{f})$, and

$$
\boldsymbol{y}(\boldsymbol{f})=L_{1} L_{2}\left[X_{1}(\boldsymbol{L} \boldsymbol{f}) e^{j 2 \pi c_{1}^{T} \mathrm{f}}, \ldots, X_{p}(\boldsymbol{L} \boldsymbol{f}) e^{j 2 \pi \mathrm{c}_{p}^{T} \mathrm{f}}\right]^{T}
$$

It can be shown [5] that the quantities $\boldsymbol{z}(f)$ and $\boldsymbol{y}(\boldsymbol{f})$ are related by

$$
\boldsymbol{y}(\boldsymbol{f})=\boldsymbol{A}_{\mathcal{K}} \boldsymbol{z}(\boldsymbol{f}), \quad \boldsymbol{f} \in \mathcal{C}_{0}
$$

where $\boldsymbol{A}_{\mathcal{K}}$ is the matrix whose entries are

$$
A_{\mathcal{K}}(r, l)=\exp \left(j 2 \pi \boldsymbol{c}_{r}^{T} \boldsymbol{L}^{-1} \boldsymbol{k}_{l}\right)
$$

If the spectral support, $\mathcal{F}$, of the input signal is known, then so are the sets $\mathcal{K}_{i}$. In this case, sampling patterns exist for which $\boldsymbol{A}_{\mathcal{K}}$ has full column rank. Universal patterns, for example, guarantee this condition. This is discussed in detail in the next section. In particular, a universal pattern corresponding to $p=q$ will yield an average sampling rate of $\frac{q}{L_{1} L_{2}}=\Omega \mathrm{L}$ which, as can be seen geometrically, can be made arbitrarily close to the measure of the set $\mathcal{F}$ (i.e. the Landau-Nyquist rate) by picking sufficiently large $L_{1}$ and $L_{2}$. We now present an improvement of the scheme just described. The idea is somewhat related to [4], but applies to 2D and has the elements of universality and spectrum blindness which [4] does not.

Let us further partition the cell $\mathcal{C}_{0}$ into $M_{1} \times M_{2}$ smaller "subcells." For the moment, we shall think of $M_{1}$ and $M_{2}$ as being large integers. The basis for choosing them will be explained later. Let the subcells 
be indexed as $\left\{\mathrm{s}_{1}, \mathrm{~s}_{2}, \ldots, \mathrm{s}_{M_{1} M_{2}}\right\}$ in an arbitrary way, e.g. lexicographically

$$
\begin{aligned}
\mathrm{s}_{M_{1} j+i+1} & =\mathrm{s}_{0}+\left(\frac{i}{M_{1} L_{1}}, \frac{j}{L_{2} M_{2}}\right) \\
\mathrm{s}_{0} & =\left[0, \frac{1}{M_{1} L_{1}}\right] \times\left[0, \frac{1}{M_{2} L_{2}}\right]
\end{aligned}
$$

for $0 \leq i<M_{1}$ and $0 \leq j<M_{2}$, is an ordering of the subcells. We define the following spectral index sets:

$$
\begin{aligned}
\mathcal{K}_{i} & =\left\{\boldsymbol{l} \in \mathcal{N}\left(L_{1}, L_{2}\right):\left(\boldsymbol{L}^{-1} \boldsymbol{l}+\mathrm{s}_{i}\right) \cap \mathcal{F} \neq \emptyset\right\} \\
& \equiv\left\{\boldsymbol{k}_{1}^{i}, \boldsymbol{k}_{2}^{i}, \ldots, \boldsymbol{k}_{q_{i}}^{i}\right\} \subset \mathcal{N}\left(L_{1}, L_{2}\right)
\end{aligned}
$$

where $q_{i}$, the cardinality of $\mathcal{K}_{i}$, represents the number of "active subcells" that overlap at $\mathrm{s}_{i}$ when the input signal is decimated by $\boldsymbol{L}$. An active subcell $\mathrm{s} \in[0,1]^{2}$ is, of course, one that is not disjoint from $\mathcal{F}$, i.e. $\mathrm{s} \cap \mathcal{K} \neq$ $\emptyset$. It can be verified, on the $i$-th subcell, that

$$
\boldsymbol{y}(\boldsymbol{f})=\boldsymbol{A}_{\mathcal{K}_{i}} \boldsymbol{z}_{i}(\boldsymbol{f}), \quad \boldsymbol{f} \in \mathrm{s}_{i}
$$

where $\boldsymbol{A}_{\mathcal{K}_{i}}\left(\right.$ size $\left.p \times q_{i}\right)$ and $\boldsymbol{z}_{i}(\boldsymbol{f})\left(\right.$ size $\left.q_{i} \times 1\right)$ are defined as

$$
\begin{gathered}
A_{\mathcal{K}_{i}}(r, l)=\exp \left(j 2 \pi \boldsymbol{c}_{r}^{T} \boldsymbol{L}^{-1} \boldsymbol{k}_{l}^{i}\right) \\
\boldsymbol{z}_{i}(\boldsymbol{f})=\left[X\left(\boldsymbol{f}+\boldsymbol{L}^{-1} \boldsymbol{k}_{1}^{i}\right), \ldots, X\left(\boldsymbol{f}+\boldsymbol{L}^{-1} \boldsymbol{k}_{q}^{i}\right)\right]^{T}
\end{gathered}
$$

If we choose a universal sampling pattern corresponding to $p=q^{\prime}\left(M_{1}, M_{2}\right) \doteq \max _{i} q_{i}$, then each of the above equations will be invertible, yielding a stable reconstruction procedure. Therefore

$$
\boldsymbol{z}(\boldsymbol{f})=\boldsymbol{A}_{\mathcal{K}}^{\dagger} \boldsymbol{y}(f)
$$

This equation relates the pieces of the $2 \mathrm{D}$ spectrum $X(f)$ to the Fourier transforms of the multi-coset samples. The reconstruction of $x(\boldsymbol{n})$ can now be accomplished using a 2D multi-rate digital filtering system [6]. The continuous function $x(\boldsymbol{t})$ can be recovered from $x(\boldsymbol{n})$ using a $2 \mathrm{D}$ sinc interpolation.

The obvious advantage this scheme offers over the previous one is the reduction in sampling rate. The average sampling rate for the scheme described is therefore equal to $q^{\prime}\left(M_{1}, M_{2}\right) / L_{1} L_{2}$. Note that, for a given spectral support $\mathcal{F}$, we have $q^{\prime}\left(M_{1}, M_{2}\right) \leq q$, since the maximum number of subcells overlapping any where (due to aliasing) cannot exceed the total number of active cells. In fact, we expect the inequality to be rather loose in many real cases. As an example consider the following rather extreme situation:

$$
\mathcal{F}=\bigcup_{i=1}^{M_{1} M_{2}}\left(\boldsymbol{L}^{-1} \boldsymbol{l}_{i}+\mathrm{s}_{i}\right)
$$

All cells are active (i.e. $\left.q=L_{1} L_{2}\right)$ at resolution $\left(L_{1}, L_{2}\right)$. Even so, the signal can be recovered from its samples taken at the theoretical minimum rate of $\frac{1}{L_{1} L_{2}}$, since the maximum number of overlaps at this resolution is $q^{\prime}\left(M_{1}, M_{2}\right)=1$. The formulation of the problem in [5] is a special case of the proposed scheme corresponding to $M_{1}=M_{2}=1$, while the one in [6] corresponds to the case $M_{1}=M_{2}=\infty$. Increasing $M_{1}$ and $M_{2}$ generally causes $q^{\prime}\left(M_{1}, M_{2}\right)$ (and hence the average sampling rate also) to decrease, though not necessarily monotonically.

\section{SPECTRUM BLIND RECONSTRUCTION}

Feng and Bresler [3, 5] demonstrate that multi-coset sampling allows reconstruction of multi-band signals without using explicit knowledge of the $\mathcal{F}$, provided that a bound on the quantity $\Omega \mathrm{L}$ at a particular resolution $\left(L_{1}, L_{2}\right)$ is known. It turns out that the same algorithm is applicable to spectrum blind reconstruction in our proposed scheme also. In the context of blind reconstruction, the following definition is useful:

Definition 1. A sampling pattern $\left\{\boldsymbol{c}_{i}\right\}$ is termed "universal" if the $p \times p$ matrix $\boldsymbol{A}_{\mathcal{K}}$ has full column rank for every subset $\mathcal{K} \subset \mathcal{N}\left(L_{1}, L_{2}\right)$ of no more than $p$ elements, i.e. $|\mathcal{K}| \leq p$.

We assume that the sampling pattern used is universal, as we shall see, since they allow spectrum blind reconstruction. The following is an important consequence of universality.

Theorem 1. Suppose $p \geq q+1$ and $\left\{\boldsymbol{c}_{i}, i=1, \ldots, p\right\}$ be a universal pattern and $\mathcal{K}_{1}, \mathcal{K}_{2} \subset \mathcal{N}\left(L_{1}, L_{2}\right)$ are such that $\left|\mathcal{K}_{1}\right|=\left|\mathcal{K}_{2}\right|=q$. Then $\mathcal{K}_{1}=\mathcal{K}_{2}$ if and only if $R\left(\boldsymbol{A}_{\mathcal{K}_{1}}\right)=R\left(\boldsymbol{A}_{\mathcal{K}_{2}}\right)$ where $R(\cdot)$ denotes the range space.

Proof. Suppose that $R\left(A_{\mathcal{K}_{1}}\right)=R\left(\boldsymbol{A}_{\mathcal{K}_{2}}\right)$. Furthermore, if $\mathcal{K}_{1} \neq \mathcal{K}_{2}$ then let $\mathcal{K}=\mathcal{K}_{1} \cup\{\boldsymbol{k}\}$ for some $\boldsymbol{k} \in$ $\mathcal{K}_{2} \backslash \mathcal{K}_{1}$. Then, clearly $R\left(\boldsymbol{A}_{\mathcal{K}}\right)=R\left(\boldsymbol{A}_{\mathcal{K}_{1}}\right)$ and hence $\operatorname{rank}\left(R\left(\boldsymbol{A}_{\mathcal{K}}\right)\right)=q$. This observation does not concur with the universality of the sampling pattern, since $|\mathcal{K}|=q+1 \leq p$. Hence $\mathcal{K}_{1}=\mathcal{K}_{2}$. The converse result needs no proof.

We shall attempt to solve (2) in two steps on each subcell. The first step is to compute the spectral index set $\mathcal{K}_{i}$ and the second is to find $\boldsymbol{z}_{i}(\boldsymbol{f})$ by solving those equations. Observe that (2) implies that $\boldsymbol{Y}(\boldsymbol{f}) \in R\left(\boldsymbol{A}_{\mathcal{K}_{i}}\right)$, for $\boldsymbol{f} \in \mathrm{s}_{i}$ and each $i$. This immediately suggests the following solution to step 1 of the 
problem

$$
\begin{aligned}
\hat{\mathcal{K}}_{i} & =\arg \min _{|\mathcal{K}|=q} \int_{\mathbf{f} \in \mathrm{S}_{i}}\left\|\boldsymbol{P}_{\mathcal{K}} \boldsymbol{y}(\boldsymbol{f})\right\|^{2} d \boldsymbol{f} \\
& =\arg \min _{|\mathcal{K}|=q} \operatorname{tr}\left\{\boldsymbol{P}_{\mathcal{K}} \boldsymbol{R}_{i}\right\}
\end{aligned}
$$

where $\boldsymbol{P}_{\mathcal{K}}=\boldsymbol{I}-\boldsymbol{A}_{\mathcal{K}} \boldsymbol{A}_{\mathcal{K}}^{\dagger}$ is the projection matrix onto $R\left(\boldsymbol{A}_{\mathcal{K}}\right)^{\perp}$ and

$$
\boldsymbol{R}_{i}=\int_{\mathrm{f}_{\in} \mathrm{S}_{i}} \boldsymbol{y}(\boldsymbol{f}) \boldsymbol{y}^{*}(\boldsymbol{f}) d \boldsymbol{f}
$$

The quantity $\boldsymbol{R}_{i}$ can be computed using a $2 \mathrm{D}$ multirate system related to the one used for reconstruction. There, however, remains the question of whether or not the minimizing solution is unique. Fortunately, we can answer that question in the affirmative:

Theorem 2. The minimization (6), almost surely has a unique solution, provided that the functions $\boldsymbol{z}_{i}(\boldsymbol{f})$ are random variables chosen according to continuous probability distribution.

Proof. Suppose that $\mathcal{K}_{a}$ and $\mathcal{K}_{b}$ (of $q$ elements each) are distinct solutions to (6), then we have

$$
\boldsymbol{y} \in R\left(\boldsymbol{A}_{\mathcal{K}_{b}}\right) \cap R\left(\boldsymbol{A}_{\mathcal{K}_{a}}\right)
$$

Meanwhile, Theorem 1 says that the $q$-dimensional subspaces $R\left(\boldsymbol{A}_{\mathcal{K}_{a}}\right)$ and $R\left(\boldsymbol{A}_{\mathcal{K}_{b}}\right)$ are different since $\mathcal{K}_{a} \neq$ $\mathcal{K}_{b}$. Therefore the intersection of these subspaces is at most $(q-1)$-dimensional, and consequently has measure zero in its $q$-dimensional parent space. Therefore the condition (8) is almost surely not satisfied, since $\boldsymbol{z}_{i}(\boldsymbol{f})$ are randomly chosen according to a continuous probability distribution.

Of course, we can always construct pathological examples, where (6) has multiple solutions. Note that (6) is essentially a best subset selection problem.

There is an issue concerning the choice of the subcell size (or equivalently the integers $M_{1}$ and $M_{2}$ ). The smaller the size of the subcell, the more sensitive (6) is to the effects of input sample noise (in terms of SNR). For larger subcells, the averaging operation in (6) improves the SNR, however, this is accompanied by an increase in the average sampling rate. There is a compromise to make between a good sampling rate (small subcells) and lower sensitivity to input noise (large subcells)

\section{APPLICATION TO FOURIER IMAGING}

In many coherent imaging modalities such as imaging by sensor array arrays or SAR as well as MRI, the available information is a set of samples of the Fourier transform, rather than the original object itself. The problem, therefore, is to recover the object from its Fourier samples, which is an exact dual of the sampling problem considered in this paper with the roles of spatial coordinates and frequencies interchanged. Our results on sampling of $2 \mathrm{D}$ signals can be directly applied to this problem.

Typically, the object itself would sparse, or can be made sparse by a linear time invariant operation. The implication is that one only needs a small fraction of Fourier samples, to reconstruct the original object. Note that the advantage of a linear time invariant operation is that the Fourier transform is multiplied by its corresponding frequency response.

\section{SIMULATION RESULTS}

The sampling and reconstruction was tested on an image, Figure 2, of spatial occupancy about $9 \%$. The test image has a resolution of $336 \times 640$ pixels, instead of being a $2 \mathrm{D}$ function of continuous spatial variables. Such an image was chosen merely for convenience. The samples of the signal are taken in the transform domain according to a multi-coset sampling scheme corresponding to $\boldsymbol{L}=\operatorname{diag}(4,5)$.

If the subcells $\mathrm{s}_{i}$ are chosen to image pixels (the smallest possible), this corresponds to $M_{1} \times M_{2}=84 \times$ 128 , or a total of 10572 subcells. Then the maximum number of overlaps on any subcell occurring due to sub-sampling turns out to be $q^{\prime}\left(M_{1}, M_{2}\right)=7$. Therefore $p=8$ is necessary for perfect reconstruction from samples. This corresponding to a sampling rate of $\frac{8}{20}=0.4$, a $60 \%$ improvement over the Nyquist rate for this signal. To approach the Landau-Nyquist rate of 0.09 , larger $L_{1}$ and $L_{2}$ can be used. Based on the sampling performance on random images, a search over 256 random patterns of length $p=8$, yielded a good pattern,

$$
\begin{aligned}
\boldsymbol{c}_{i} & =\left(\gamma_{i} \bmod 4, \gamma_{i} \bmod 5\right) \\
\left\{\gamma_{i}\right\} & =\{1,2,8,10,15,16,17,19\}
\end{aligned}
$$

which was used in all simulation experiments. White noise of known variance was added to the Fourier samples in all simulations. This is a reasonably good model that account for nonidealities in the system acquiring the samples. This may be incorporated into (2) by adding, to its right-hand side, a noise vector $\boldsymbol{n}(\boldsymbol{f})$ satisfying $E\left(\boldsymbol{n}(\boldsymbol{f}) \boldsymbol{n}\left(\boldsymbol{f}^{0}\right)\right)=\sigma^{2} \delta\left(\boldsymbol{f}-\boldsymbol{f}^{0}\right)$. The spectral sets $\mathcal{K}_{i}$ are found by solving (6) on each subcell. Since this subset selection problem is NP complete [7], a combination of a greedy algorithm and an exhaustive search (for the case when the greedy algorithm returns too large a residual in (6)) was used. Figures 2 and 3 show the original image and reconstructed image when the input SNR is $41 \mathrm{~dB}$. The algorithm failed to compute 
the correct $\mathcal{K}_{i}$ in $0.16 \%$ of the cases, due the presence of the input noise. The corresponding output SNR was $22 \mathrm{~dB}$.

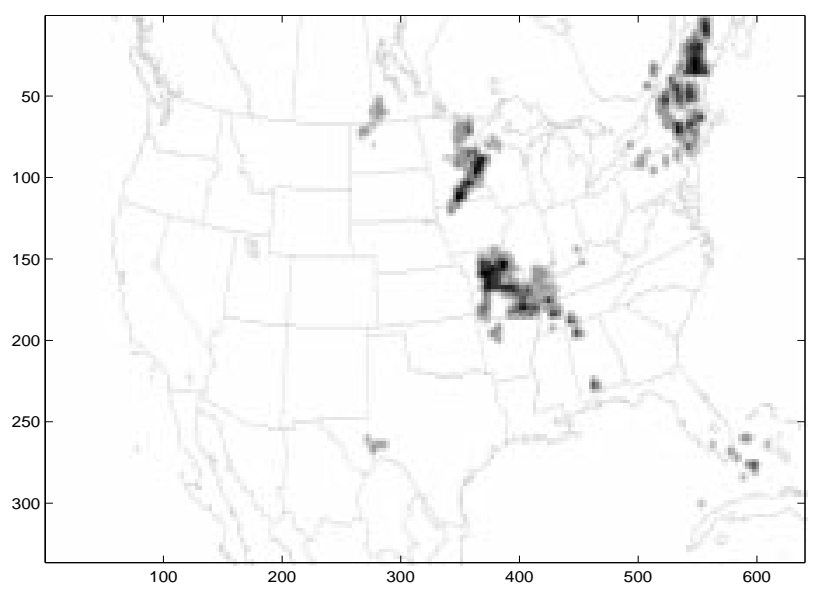

Figure 2: Sparse test image: radar map of the US

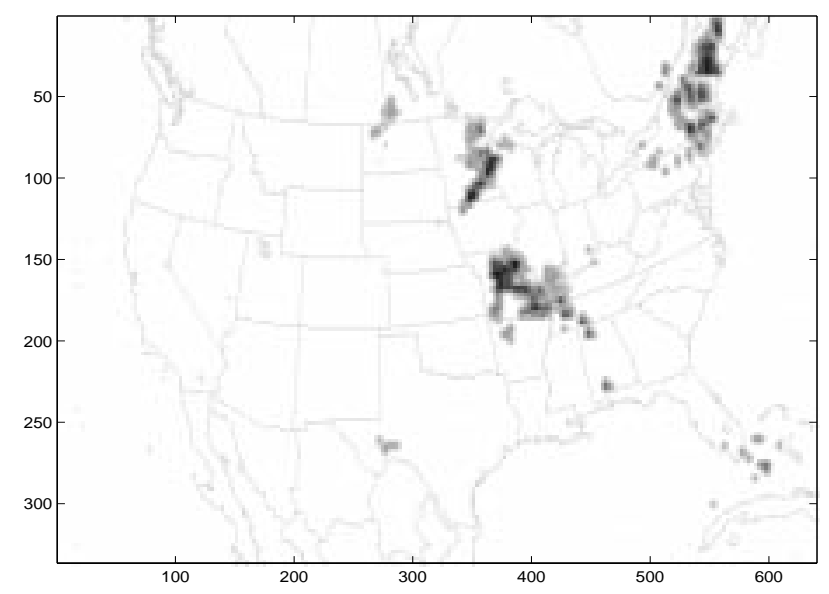

Figure 3: Image reconstructed from the samples of the noisy test image.

The effect of changing the subcell size was studied for a fixed input SNR of $21 \mathrm{~dB}$. The subset selection failure rates and the output SNR's are tabulated in Table 1. These simulations used a plain greedy algorithm, which generally produces about $1 \%$ subset selection errors even in the no noise case. An exhaustive search to solve (6) is likely to produce better results. Among the cases considered in Table 1 , the optimal subcells are blocks of $2 \times 2$ pixels. As the subcell size is increased from its smallest size of 1 pixel, there is an improvement in output SNR. But, for larger subcells, the condition $q_{i} \leq p-1$ is violated more often for a fixed $\mathrm{p}$, and this eventually degrades the output SNR.

\section{REFERENCES}

[1] K. Cheung and R. Marks, "Image sampling below the Nyquist density without aliasing," J. Opt. Soc. Am. A, vol. 7, pp. 92-105, 1990.

[2] P. P. Vaidyanathan and V. Liu, "Efficient reconstruction of band-limited signals from nonuniformly decimated versions by use of polyphase filter banks," IEEE Trans. ASSSP, vol. 38, pp. 19271936, Nov. 1990.

[3] P. Feng and Y. Bresler, "Spectrum-blind minimumrate sampling and reconstruction of multi-band signals," in Proc. IEEE Int. Conf. ASSP, Atlanta, GA, IEEE, May 1996.

[4] C. Herley and P. W. Wong, "Minimum rate sampling of signals with arbitrary frequency support," in Proc. IEEE Int. Conf. Image Proc., Lausanne, Switzerland, IEEE, Sept. 1996.

[5] Y. Bresler and P. Feng, "Spectrum-blind minimumrate sampling and reconstruction of 2-D multi-band signals," in Proc. IEEE Int. Conf. Image Proc., Lausanne, Switzerland, IEEE, Sept. 1996.

[6] R. Venkataramani and Y. Bresler, "Sub-Nyquist sampling of multiband signals: perfect reconstruction and bounds on aliasing error," in Proc. IEEE Int. Conf. ASSP, Seattle, WA, IEEE, April 1998.

[7] B. K. Natarajan, "Sparse approximate solutions to linear sytems", SIAM Journal on Computers, vol. 24, pp. 227-234, April 1995.

\begin{tabular}{|c|c|c|}
\hline Subcell Size & Failure Rate & Output SNR \\
\hline \hline $1 \times 1$ pix. & $2.93 \%$ & $13.94 \mathrm{~dB}$ \\
\hline $2 \times 2$ pix. & $1.29 \%$ & $17.46 \mathrm{~dB}$ \\
\hline $3 \times 4$ pix. & $1.59 \%$ & $14.62 \mathrm{~dB}$ \\
\hline $7 \times 8$ pix. & $8.95 \%$ & $9.13 \mathrm{~dB}$ \\
\hline
\end{tabular}

Table 1: Subset selection failure rate and output SNR for various subcell sizes. The input SNR was kept fixed at $21 \mathrm{~dB}$ 